\title{
On the Equilibrium of Pricing Assignment for Heterogeneous Wireless Access Networks
}

\author{
Carmen López $^{\dagger}$, Johnny Choque ${ }^{\dagger}$, Ramón Agüero ${ }^{\dagger}$, \\ Joan Serrat ${ }^{\ddagger}$, and Luis Muñoz ${ }^{\dagger}$ \\ ${ }^{\dagger}$ Universidad de Cantabria, Santander, Spain \\ ${ }^{\ddagger}$ Universidad Politécnica de Catalunya, Barcelona, Spain \\ $\dagger\left\{\right.$ clopez, jchoque, ramon, luis\}@tlmat.unican.es, ${ }^{\ddagger}$ serrat@tsc.upc.edu
}

\begin{abstract}
Next generation networks and service providers are rapidly evolving in order to satisfy the demands of an increasing number of users. Nowadays, one of the most relevant research lines in the Heterogeneous Wireless Access Networks realm is the use of procedures and mechanisms so as to provide intelligence to the network, with the main goal of optimizing its performance. Furthermore, mobile communication users are becoming more demanding, and thus it becomes essential for the providers to be able to offer a competitive value for money. This paper pursues the objective of obtaining, from an analytical perspective, the optimum price assignment strategy according to the characteristics of a particular scenario, analyzing the improvement attained with such optimum pricing policy as compared to a more traditional one. Game theory techniques, which are gathering the interest within the communications scientific community, are used for the analysis.
\end{abstract}

Key words: pricing, game theory, heterogeneous networks.

\section{Introduction}

In the latest years we have seen a significant evolution of the wireless communication realm, embracing both the subjacent technologies as well as their operation and management. In addition, this has come together with an increase of the user's degree of responsibility when selecting a network to connect to.

During the early days of telephony, the market was a monopoly. However, this situation has remarkably evolved and new operators have appeared, turning the market into a very competitive environment. In such scenario, operators must compete amongst them in different aspects, such as the services which are offered to the users, the corresponding Quality of Service (QoS) and/or prices. It goes without saying that, from the users' perspective, one of the most significant elements is the price to be paid. At the time of writing, the most common pricing policy is the flat-rate, where the user pays a monthly fee in exchange of various services. However, the emergence of new operators has led to a situation where churning (i.e. change between operators) needs to be considered. One potential solution to this problem is to better accomodate the pricing models, so that 
they are more suitable to the new scenarios. In this sense, it is now believed that modulating the offered price depending on the particular context might be sensible; it is worth saying that with context we refer to a relatively large number of aspects, such as the number of operators sharing the same coverage area, their capacity, number of users demanding connectivity, etc. Another assumption is that the end-user would select the cheapest alternative ${ }^{1}$. The main goal is to increase operators' benefit while not damaging (or even improving) users' satisfaction.

Besides, one tool which has recently gained a lot of relevance in the wireless communication research area is game theory. It is mainly used to analyze the behavior of different techniques, mechanisms or procedures, when various entities have conflicting interests and it usually has the goal of obtaining the best-theycan-do. In this work we propose analyzing (by means of game theory) various price assignment policies to be used by wireless Access Elements (AEs). We will obtain the potential strategies which can be established by each of the AEs, deriving the benefits associated to each of them. From those, we will propose a non-cooperative game, which will be solved so as to obtain the Nash Equilibrium Point. Finally, and to demonstrate the benefits of using this optimal strategy, we will compare the corresponding performances (in terms of the operators' benefit) with those which result when the AEs use a naive policy, establishing a fixed price.

There are some works which have previously addressed (partially) the combination of pricing, wireless heterogeneous networks and game theory. In [5] Niyato and Hossain depict various pricing-based situations over networks where only one wireless technology is available. In addition, they propose two models for price competition in a heterogeneous scenario, formulating two non-cooperative games: a simultaneous-play, whose solution is the Nash Equilibrium Point, and a leader-follower game, which is solved with the Stackelberg equilibrium. The same authors consider, in [6], the case where providers cooperate, posing a simultaneous game and introducing a scheme for revenue sharing between the service providers, based on a N-person coalition game.

Another approach to tackle the pricing problem is to analyze the situations from the point of view of the players involved in the game. Using that approach some works consider both operators and users, while others only focus on the operators. Illustrative examples of the first group are [7] and [8]. The first one mainly proposes a theoretical non cooperative game to investigate the conflict between wireless service providers and users for varying QoS requirements. On the other hand, the authors of [8] develop a framework to analyze service providers' strategies to attract users, by offering competitive prices. As a combination of these two approaches, [2] presents both non-cooperative and cooperative games, to tackle optimal pricing and network selection, respectively.

\footnotetext{
${ }^{1}$ This is actually a simplification and we could add as well more parameters into the decision process; however, by limiting this study to price, we will be able to focus on the impact that these new pricing policies might have on system performance.
} 
Finally, it is also worth mentioning that there exist other works addressing pricing policies, but without game theory. Amongst these, we could highlight [1], where the authors analyze (in general) service management policies, including pricing, which are used by the operators to maximize their benefit; in addition, they also analyze various access selection procedures, emphasizing the role of the price to pay for a connection.

In this context, the most relevant novel contributions of this work can be summarized as follows.

- Analysis of price strategies where the operators can only establish a discrete and finite set of fees.

- Deploy heterogeneous network deployments, comprising access elements with different capacities and coverages.

- Use of discrete load units, bounding the capacity of the access elements, which can not accept more connections than their available capacity.

- An extensive performance analysis, comprising different scenarios and multiple instances of them.

The paper is structured as follows. Section II presents a theoretical analysis, which aims at modeling the dynamic pricing situation and settles the rationale for the rest of the paper. The network model and the game which is posed are depicted in Section III, while Section IV describes how the game was solved. The achieved results are discussed in Section V, which identifies the benefits brought about by the optimum strategies. Finally, Section VI concludes the paper, advocating some items which are left for future work.

\section{Analysis of Dynamic Pricing Schemes}

In this work we consider a network scenario over a squared area where a set of $N$ AEs, characterized by their capacity and coverage, are deployed. In this environment, each $\mathrm{AE}$ wants to maximize its benefit; this is to say, serve as many users as possible. In order to achieve this goal, the AEs aim to motivate/encourage the users to connect to it, even if they have more alternatives.

From the point of view of the users, the motivation to select one of the options over the others is the corresponding price ${ }^{2}$. In this sense, users will select the cheapest alternative, from the available ones. Note that we further assume that any available connection alternative (provided it has enough capacity) would fulfil the requested QoS level.

Starting from the previous assumptions, and using a simple scenario, this section aims at identifying the strategy which each of the operators shall use, provided that they are aware of the rational behavior of the users, which only depends on the price offered by the AEs. We will study two different pricing policies, namely legacy/conventional and novel/dynamic. In the former scheme,

\footnotetext{
${ }^{2}$ Throughout this work we will restrict to situations in which users base their decisions only on price.
} 
AEs do not change the offered fee, while in the dynamic policy, AEs are able to adapt their fees according to the particular context, entailing them to implement various strategies to attract more users. We will assume two particular scenarios, whose results could be extended to more complex environments: one in which there is one traditional AE competing with a novel one and another one in which two AEs are able to modify their fees.

\subsection{Dynamic Vs. Traditional Pricing Policies}

First of all, lets assume that the traditional operator establishes a generic and fix price $P_{t}$, while the non-conventional operator is able to settle any price within a particular set. We can thus define a vector $\mathrm{P}=\left\{p_{1}, p_{2}, \ldots p_{t-1}, p_{t}, \ldots p_{m}\right\}$, sorted in ascending order, where $p_{t}$ equals the fee fixed by the traditional operator and $p_{m}$ is the maximum price allowed by the market. We also suppose that these prices are per user and connection (this is, they are average prices per service).

The analysis is carried out over the scenario shown in Figure 1, comprising two AEs, each belonging to any of the two aforementioned operators, with an overlap area where they try to attract as many users as they can afford. As can be seen, there exist three different areas: A (only covered by the non conventional operator), $\mathrm{B}$ (covered by the two $\mathrm{AE}$ ) and $\mathrm{C}$ (in which the only access alternative is the traditional operator); we define $u_{A}, u_{B}$ and $u_{C}$ as the number of users within each of these areas, respectively. Under these circumstances, the users who are within the non-overlap areas can be seen as preferred users for the corresponding operator (the one which covers such area). This assumption is sensible, since they would surely select such operator (being the only access alternative).

In this sense, once $u_{A}$ and $u_{C}$ have already connected to the corresponding AEs, the two operators can offer the rest of their resources, $C_{t}$ and $C_{n c}$ for the traditional and non-conventional operators, respectively, to those users within

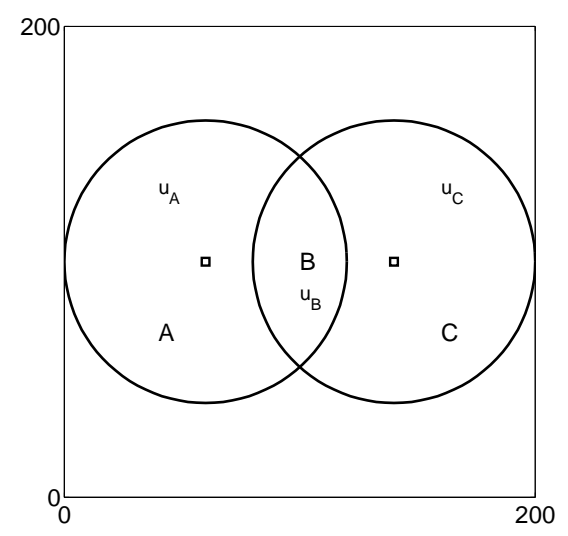

Fig. 1. Scenario used for the analysis. 
the overlap area. Three different situations can be identified, depending on the available resources for each of the AEs, as depicted below.

1. $C_{t}=C_{n c}$

We will focus on the benefit obtained by the non-conventional operator, represented in terms of the number of users within area B $\left(u_{B}\right)$. In this case, as can be seen in Figure 2(a), we can further distinguish two possibilities:

- The non-conventional operator assigns a smaller price than the traditional one (in this analysis we will assume that it sets the maximum fee fulfilling such requirement, i.e. $p_{t-1}$. As can be seen in Figure 2(a), the users will therefore select the non conventional operator and therefore the benefit equals $\left(u_{B} \cdot p_{t-1}\right)$. Furthermore, the maximum achieved benefit will be bounded by the AE's capacity, $\left(C_{n c} \cdot p_{t-1}\right)$. In this sense, if the number of users is greater than the non conventional operator resources, some of them would select the traditional alternative.

- The non-conventional operator establishes a price higher than $p_{t}$, (in this case, it fixes the maximum one, i.e. $p_{m}$ ). Since it provides a cheaper alternative, users would select the traditional operator, but when its available resources are fewer than the number of users, the rest of users would choose the non conventional operator.

The upper line in Figure 2(a) represents the maximum benefit that the nonconventional operator can obtain for each value of $u_{B}$. It is worth highlighting $u_{x}$, which determines the decision threshold (in the number of users) for deciding upon the fee to establish. This value is the point on which the two aforementioned benefits are the same, an can be calculated as follows:

$$
\left(u_{x}-C_{t}\right) \cdot p_{m}=C_{n c} \cdot p_{t-1} \rightarrow u_{x}=\frac{p_{t-1}}{p_{m}} \cdot C_{n c}+C_{t}
$$

Hence, the intervals and the optimum prices to be used are:

$$
\begin{gathered}
u_{B}<u_{x} \Rightarrow P=p_{t-1} \\
u_{B}>u_{x} \Rightarrow P=p_{m}
\end{gathered}
$$

2. $C_{t}<C_{n c}$

As happened before, we can also distinguish two cases, depending on the point where the benefit functions of the non-conventional operator get the same value.

- If $\left(u_{x}>C_{n c}\right)$. The analysis is depicted in Figure 2(b) (and is similar to the one described for the former case) and the intersection point is calculated as follows.

$$
\left(u_{x}-C_{t}\right) \cdot p_{m}=C_{n c} \cdot p_{t-1} \Rightarrow u_{x}=\frac{p_{t-1}}{p_{m}} \cdot C_{n c}+C_{t}
$$

- If $\left(u_{x}<C_{n c}\right)$. This case is also shown in Figure 2(c), and the corresponding intersection point can be derived as follows.

$$
\left(u_{x}-C_{t}\right) \cdot p_{m}=u_{x} \cdot p_{t-1} \Rightarrow u_{x}=\frac{C_{t} \cdot p_{m}}{p_{m}-p_{t-1}}
$$


3. $C_{t}>C_{n c}$

Following a similar analysis as before we can obtain the benefit shown in Figure 2(d), and the point in which the non-conventional operator shall shift its strategy $\left(u_{x}\right)$ is calculated as follows.

$$
\left(u_{x}-C_{t}\right) \cdot p_{m}=C_{n c} \cdot p_{t-1} \Rightarrow u_{x}=\frac{p_{t-1}}{p_{m}} \cdot C_{n c}+C_{t}
$$

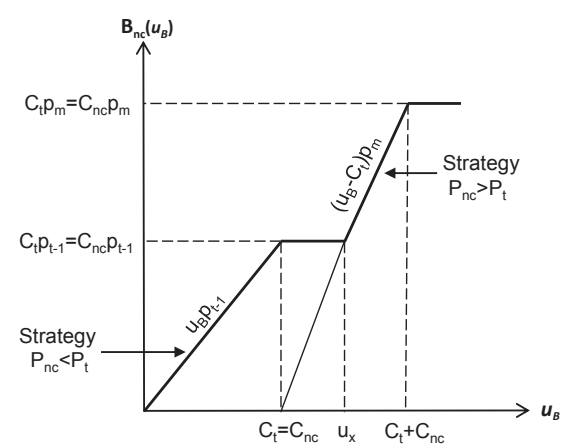

(a) Case $C_{t}=C_{n c}$

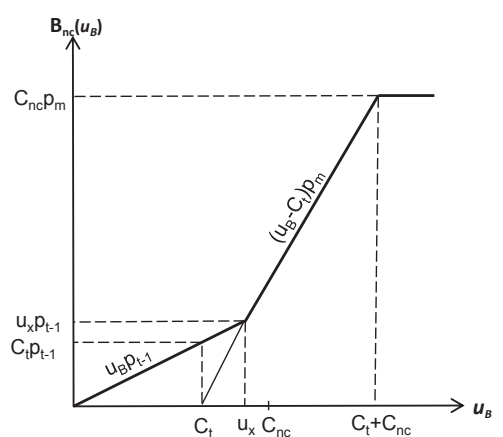

(c) Case $C_{t}<C_{n c}$ and $u_{x}<C_{n c}$

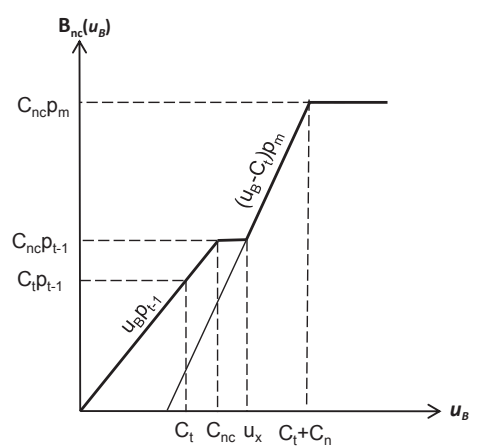

(b) Case $C_{t}<C_{n c}$ and $u_{x}>C_{n c}$

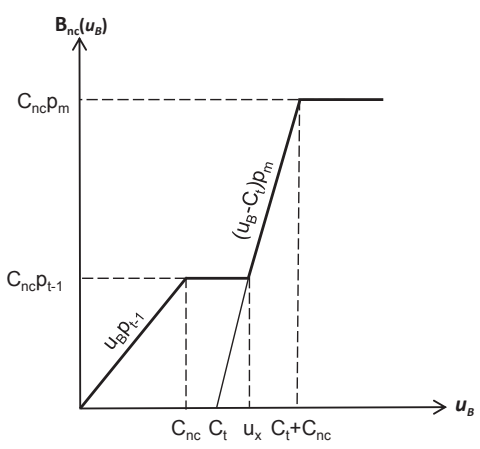

(d) Case $C_{t}>C_{n c}$

Fig. 2. Benefit of the non conventional operator against the number of users in the overlap area for the different cases

\subsection{Two Dynamic Pricing Strategies}

In this situation, both operators use dynamic pricing, and we also assume that none of them knows, a priori, the price which the other one assigns. For the analysis, we will study the behavior of the operators for all the potential combinations of prices, so as to select the one which leads the highest benefit. The scenario will be the same as the previous one, although we will have additional assumptions so as to limit the complexity of the analysis. 
- The two AEs have the same capacity.

- We will use three illustrative use cases, depending on the percentage of users which are within the overall area; in particular we will assume that these are $80 \%, 50 \%$ and $20 \%$ of the overall number of users.

For each of the three aforementioned situations we have obtained the benefit for each of the two AEs as we increase the overall number of users studying two complementary situations, which are explained below.

- The benefit which is achieved when the two AEs use dynamic pricing, but we prioritize $A E_{1}$, forcing $A E_{2}$ to establish the fee which maximizes $A E_{1}$ benefit (Figure 3(a)).

- For comparison purposes we will also assume that $A E_{2}$ uses a fixed fee, while $A E_{1}$ is able to modulate its price, as was discussed in the analysis presented before (Figure 3(b)).

From the results presented in Figure 3(a) some interesting conclusions can be derived. First of all, if the overlap area is small, we can see that the benefits of the two AEs are almost alike. The reason is that becomes more sensible for both operators to establish the average price and share the users evenly. If any of the two operators assigns the maximum price, a lower benefit would be assessed, since users would select the cheapest operator. If the AEs establish the cheapest fee, this would encourage the users within the overlap area, but globally it would lead to a lower benefit from those areas where users can only access one AE. Besides, when the overlap areas is bigger, $A E_{1}$ obtains a higher benefit. Furthermore, we can also see that the benefits of both operators get equal only when the number of users is large enough so as to allow them establishing the highest fee and get their capacity fully occupied.

On the other hand, Figure 3(b) shows the benefits which were obtained when one of the operators uses a static fee. Again, if the percentage of users within the overlap areas is small, the benefits are almost the same. Besides, when the size of

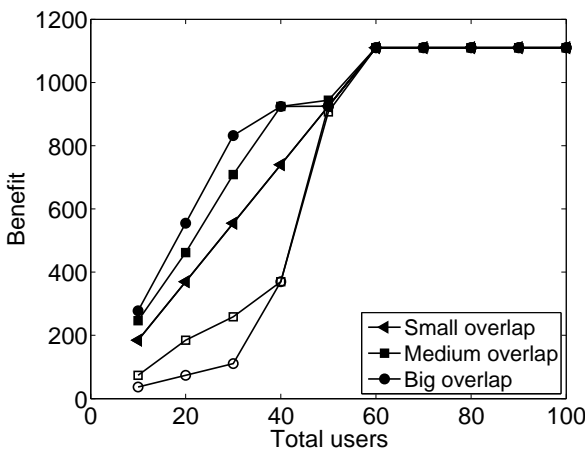

(a) Two operators with dynamic pricing

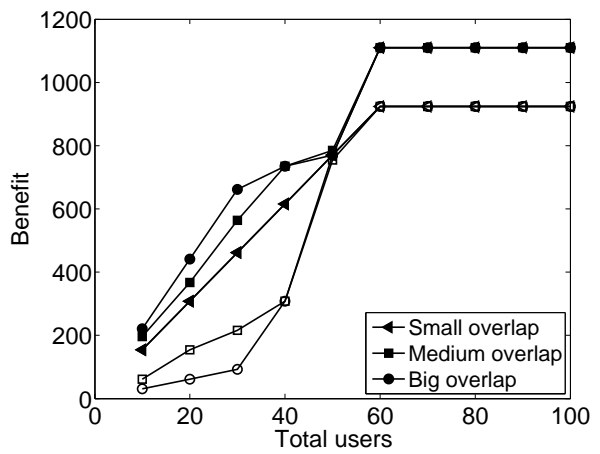

(b) One operator with dynamic pricing

Fig. 3. Overall benefit for $A E_{1}$ and $A E_{2}$. Solid markers represent the benefit obtained by the $A E_{1}$, while the empty ones are for $A E_{2}$. 
the overlap area gets higher, we can see that the operator assigning the optimum strategy gets higher benefits. In this case, since the traditional operator does not change the established fee, it never uses the highest price and, thus, its benefit would not equal the one obtained by the dynamic operator, as it happened in the previous case.

To conclude, it can be said that the dynamic pricing leads to a higher benefit. As can be seen, a thorough analysis of all the situations might be too complex to achieve (especially if we increase the number of overlap areas, AEs, etc); in order to solve these situations we will use game theory techniques, as explained in the following sections.

\section{Network Model and Non Cooperative Game}

In this section we bring about a more realistic approach for assessing the benefits of using dynamic pricing strategies. We consider a square area in which there exist $N$ access elements, which are characterized by their coverage and capacity, $\mathrm{AE}=\{1,2, \ldots, N\}$. Their position within the scenario under analysis will be done according to two different models: random and deterministic. The position of AEs and end-users leads to the definition of a set of $m$ areas, result of the overlapping of the various AEs coverage, $\Gamma=\{1,2, \ldots, m\}$.

We assume that each $\mathrm{AE}$ has a particular capacity, for which a generic and discrete unit will be used, the so-called Traffic Unit (TU) [3], no matter it refers to code, sub-carriers, time slots, etc. Anytime an end-user connects to a particular $\mathrm{AE}$, one of its resources is consumed, and thus they could eventually get extinguished.

Furthermore, we also need to establish the way an end-user selects an AE, whenever she has different connection alternatives; in the framework of this work, we have considered that the users will choose the $\mathrm{AE}$ which offers the best price between the available choices, and if there are various AEs with the same price the users selects one randomly.

Considering the presented network model, we introduce herewith the noncooperative game which will allow the operators to find the optimum price to impose. It is assumed that the players are the AEs which are deployed within the scenario and that their strategy spaces will be determined by the various possibilities they have so as to assign a price from their possibilities; thus, $\left(x_{i}\right)^{j}$ would represent the prices that the $i^{\text {th }} \mathrm{AE}$ assigns to all areas under its coverage in the $j^{\text {th }}$ strategy.

Furthermore, the overall set of strategies can be obtained as the cartesian product of the individual ones (per AE).

$$
\mathbb{X}=\mathbf{x}_{1} \times \mathbf{x}_{2} \times \ldots \times \mathbf{x}_{N}
$$

Hence, we can define a strategy profile ${ }^{3}(s)$ as an element of the space strategy

\footnotetext{
${ }^{3}$ We will use the words strategy and strategy profile indifferently.
} 


$$
s \in \mathbb{X}, s=\left(s_{1}, s_{2} \ldots s_{N}\right) \text { with } s_{i} \in \mathbf{x}_{i}
$$

For each of the strategy profile, we obtain the benefits for all AEs and, after that, we can finally pose the finite game to be solved. The corresponding maximization problem can be strictly set out to maximize the following expression:

$$
B_{i}=\sum_{\forall k: i \in k} \overline{\left(b_{i}\right)_{k}}
$$

where $\overline{\left(b_{i}\right)_{k}}$ would be the average expected benefit by the $i^{\text {th }} \mathrm{AE}$ in the $k^{\text {th }}$ area.

For the specified selecting model, the average number of users selecting an $\mathrm{AE}$ will depend on both the price such $\mathrm{AE}$ assigns and the fees fixed by the rest of AEs. As mentioned before, if various AEs assign the same price and they are giving coverage at the same area, users will make their choice randomly, so the AE more likely to be selected is that which has more available resources within such area ${ }^{4}$.

There exits three situations which bring about different benefits, as discussed below.

1. Case $p_{i}<p_{t} \forall A E_{t} \in k$

$$
\overline{\left(b_{i}\right)_{k}}=\left\{\begin{array}{lll}
u_{k} \cdot p_{i} & \text { if } & u_{k}<r_{i} \\
r_{i} \cdot p_{i} & \text { if } & u_{k}>r_{i}
\end{array}\right.
$$

where $p_{i}$ and $r_{i}$ are the price assigned and the available resources of $\mathrm{AE}_{i}$, respectively, and $u_{k}$ are the users deployed in the area $k$. When $\mathrm{AE}_{i}$ has the lowest price of all the connection alternatives within such area, the users would select $\mathrm{AE}_{i}$ until all its resources are exhausted.

2. Case $p_{i}>p_{t} \forall A E_{t} \in k$

$$
\overline{\left(b_{i}\right)_{k}}=\left\{\begin{array}{lll}
\left(u_{k}-r_{j}\right) \cdot p_{i} & \text { if } \quad\left(u_{k}-r_{j}\right)<r_{i}, \sum_{\forall j \neq i: j \in k} r_{j}<u_{k} \\
r_{i} \cdot p_{i} & \text { if } \quad\left(u_{k}-r_{j}\right)>r_{i}, \sum_{\forall j \neq i: j \in k} r_{j}<u_{k} \\
0 & \text { if } \quad \sum_{\forall j \neq i: j \in k} r_{j}>u_{k}
\end{array}\right.
$$

where $\sum_{\forall j \neq i: j \in k}$ is the overall resources from all AEs available in the $k_{t h}$ area with a lower price than $\mathrm{AE}_{i}$. In this case, the price established by $\mathrm{AE}_{i}$ is higher and thus, users would only select it if the resources from the rest of alternatives is not enough for satisfying all users in the area.

\footnotetext{
${ }^{4}$ We do not assume that AEs are limiting their resources within a particular area, since this would change the strategy space, by adding another dimension to the strategy profile, but we refer to the remaining resources once the users within the single areas (only covered with an AE) have already established their connectivity.
} 
3. Case $p_{i}=\min p_{t} \forall A E_{t} \in k$

$$
\overline{\left(b_{i}\right)_{k}}= \begin{cases}\frac{r_{i}}{\sum_{\forall j: j \in k} r_{j}} \cdot u_{k} \cdot p_{i} & \text { if } u_{k}<\sum \forall j \neq i: j \in k \\ r_{i} \cdot p_{i} & \text { if } u_{k}>\sum_{\forall j: j \in k}\end{cases}
$$

In this case the price of $\mathrm{AE}_{i}$ is the minimum one within the area, but there are more AEs which have establihed such fee. In this case the benefit obtained (users would select one alternative on a random way) depends on the number of resources offered by $\mathrm{AE}_{i}$, as compared with those offered by the AEs which established the same price.

We could also think on a situation merging cases 2 and 3, when two or more AEs establish the same price, but it is not the minimum amongst those offered in such area, but the demand can not be satisfied by those access alternatives (the cheapest AEs).

Once we have obtained the benefits, we can pose the Price Assignment Game $(P A G)$ as follows, where $s$ is a strategy profile:

$$
P A G=\left\{N, \mathbb{X},\left\{b_{i}(s)\right\}_{i \in N}\right\}
$$

The following definition (see e.g. [9] for a deep treatment of Game Theory), establish the solution of the corresponding problem.

Definition 1 A strategy profile $s$ is a pure Nash Equilibrium if

$$
b_{i}\left(s_{i}, s_{-i}\right) \geq b_{i}\left(s_{i}^{\prime}, s_{-i}\right)
$$

for all $s_{i}^{\prime} \in S$ and each player $i$, where $s_{-i}=s_{1}, s_{2}, \ldots s_{i-1}, s_{i+1}, \ldots s_{n}$.

\section{Implementation}

In order to solve the aforementioned problem, two separated phases are followed: the first one (getStrategies) establishes the strategy space, sets out the corresponding game and obtains its Nash Equilibrium, while the second one (compareStrategies) studies the benefits which are brought about by such optimum solution as compared to a situation where the AEs always assign a fixed price.

Both phases share some common steps, which are briefly described below.

1. Scenario setup. Both AEs and end-users are deployed within the area under analysis. Random as well as deterministic deployments are supported. For the compareStrategies, only deterministic positioning is used, since the scenario must be the same as the one which was used during the getStrategies.

2. Connectivity. Once end-users and AEs are deployed within the scenario, we establish the existing links between them (whenever an end-user lies within the coverage area of a particular AE.). 
3. Area establishment. In the framework of this work, an area is defined by a group of users which might be able to connect to the same group of AEs. Furthermore we can distinguish between two types of areas: single and overlap areas; the former group corresponds to those areas where there is only one reachable $\mathrm{AE}$, while for the second group more than one $\mathrm{AE}$ would be reachable.

After these common procedures, the execution for the getStrategies and compareStrategies differs.

In the case of the getStrategies we implemented some functions so as to identify all possible strategies and, afterwards, process them to obtain the benefit for each $\mathrm{AE}$, with the goal of finding the optimum strategy. The concrete steps which are undertaken are briefly depicted below.

1. Getting strategies. The first step is to actually obtain the strategy space; strategies are established for each of the AEs. Each AE will have as many strategies as prices it can assign. For obtaining our results AEs can impose three prices but it can be assigned as many prices as we want. Finally, to find the overall set of strategies, we obtain the combinations which can be established from the strategies of all AEs applying the cartesian product.

2. Obtain benefits. Once the strategy space has been obtained, we calculate the average benefit obtained by the AEs for each strategy. For this purpose, strategies are processed so as to derive the corresponding benefit in each one of the areas applying equations 10, 11 or 12 depending on the situations. Finally we accumulate all the benefits over those areas for every AE.

3. Solve the game. In this point, having in mind the strategies and their benefits, we can already pose a finite game to be solved. The pure Nash Equilibrium Point (NEP) is obtained with the Gambit software [4].

Regarding the compareStrategies method, the goal is to analyze the benefits which would be obtained by imposing the optimum strategy (i.e. using the previously found NEP in the getStrategies procedure), comparing them with those which would be achieved when the AEs use the medium price. In either of the two cases, we go through all the end-users, who use the aforementioned connecting decision (until they establish a connection or they do not have any available possibility). The order in which end-users are processed has a clear impact on the resulting connectivity, so we randomly shuffle them, repeating the same process for a sufficiently big number of times.

\section{Discussion of results}

In this section, we present and discuss the results which were obtained by implementing the procedure which was previously depicted. We assume a squared area (200 $m$ side) in which we deploy 7 different AEs, belonging to three types of Radio Access Technology (RAT), whose characteristics are shown in Table 1. 
Note that for $\mathrm{RAT}_{1}$ we fix a sufficiently large coverage so as to ensure that it always cover the complete scenario, no matter the position of the $\mathrm{AE}$ is. We consider two complementary network deployments: deterministic and random; the topology of the former can be seen in Figure 4(a), while Figure 4(b) shows an illustrative example of a random deployment.

We increase the number of users from $10 \%$ to $200 \%$ of the overall network capacity (i.e. from 7 to 130 users) and for each case we execute 100 independent analysis (so as to ensure the statistical validity of the results). For the random deployment, both AEs, as well as end-users' positions, are changed every time, while for the deterministic case, it is only the users' position which changes in all runs. In all cases we compare the results achieved between the NEP and the naive strategy (the latter corresponding to the situation in which AEs assign the medium price).

First, Figures 5(a) and 5(b) show the benefit achieved by the whole network. The better distribution of AEs in the deterministic topology is clearly reflected in the obtained results, since the benefit by the overall network is always larger in this case. Comparing both network topologies, we can see that the greater gain is achieved when the number of users equals the network capacity, being this difference lower in the edge cases. The gain which the NEP strategy yields is also more relevant for the deterministic scenario, being around 36\% when

Table 1. Types of RAT used during the analysis

\begin{tabular}{|c|c|c|c|}
\hline \multicolumn{3}{|c|}{ RAT Range Capacity } & \multirow{2}{*}{$\begin{array}{c}\text { \# of AEs } \\
\text { in the scenarios }\end{array}$} \\
\hline$I D$ & $(m)$ & (TU) & \\
\hline 1 & full & 20 & 1 \\
\hline 2 & 50 & 10 & 3 \\
\hline 3 & 30 & 5 & 3 \\
\hline
\end{tabular}

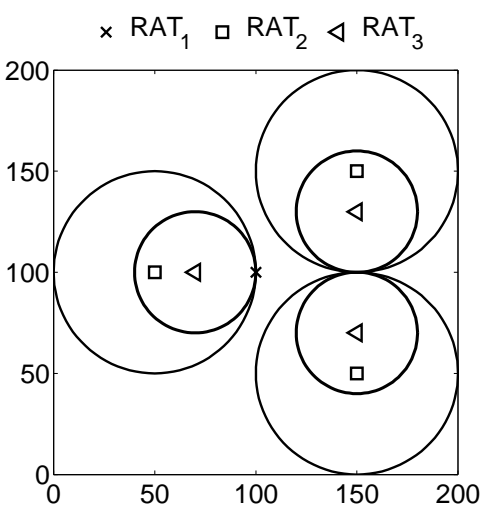

(a) Deterministic

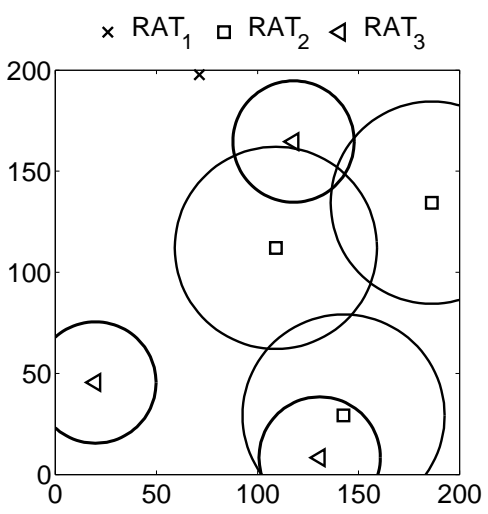

(b) Random (illustrative example)

Fig. 4. Network topologies used during the analysis. 

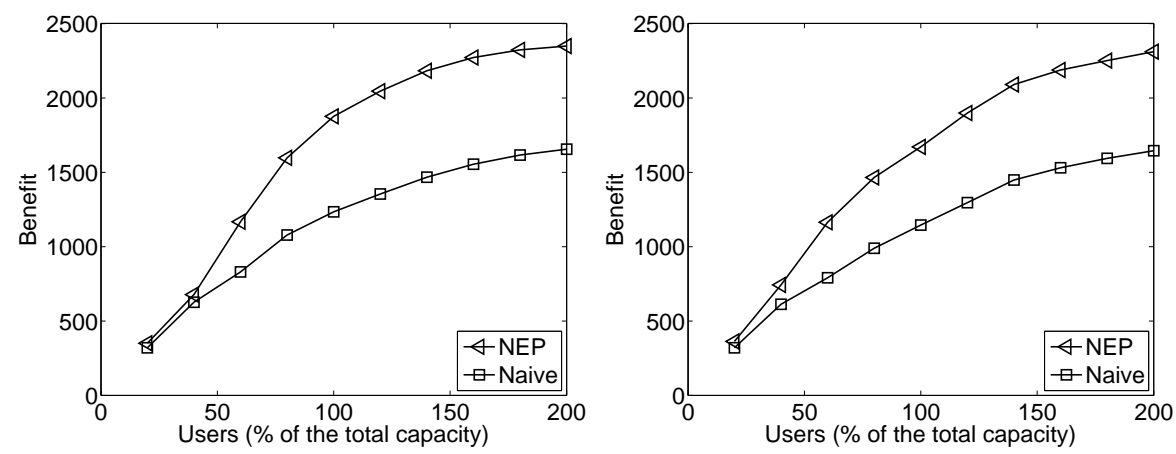

(a) Overall benefit for the deterministic (b) Overall benefit for the random scescenario nario
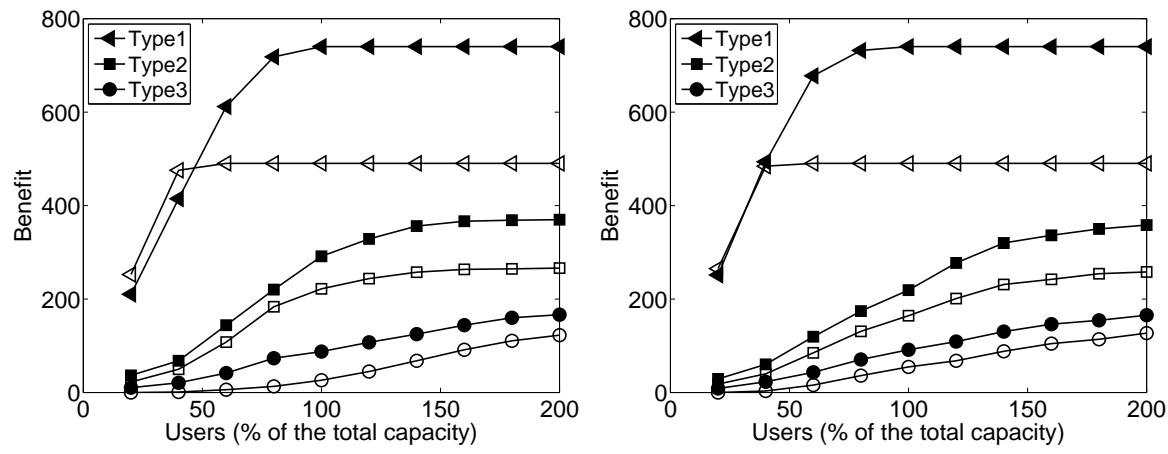

(c) Benefit per RAT in the deterministic (d) Benefit per RAT in the random scescenario nario

Fig. 5. Overall benefit and benefit per RAT type for the two scenarios. Solid markers are the ones obtained with the NEP strategy, and the empty ones are those corresponding to the naive assignment.

the offered load (number of users) equals the overall network capacity. Another interesting aspect is that there is not any particular gain when the offered load is low (end-users are below $40 \%$ of the overall network capacity).

In order to get a better understanding of the differences between the NEP and the naive strategies, Figures 5(c) and 5(d) depict the benefit for the three RAT types which are being used (we still use the same two network deployments). First of all, it is worth highlighting that the highest capable RAT ( $\left.\mathrm{RAT}_{1}\right)$ gets the greater gain after applying the NEP strategy, but for situations in which users are fewer than the $40 \%$ of the network capacity, where the benefit is very similar (or even slightly worse). Comparing both network topologies, it can be seen that the results are rather similar for $\mathrm{RAT}_{1}$. On the other hand, for both $\mathrm{RAT}_{2}$ and $\mathrm{RAT}_{3}$, we can see the consequences of having a better deployment, 
since the gain is, in most cases, higher than the one assessed in the random scenario.

\section{Conclusions}

In this work we have proposed a dynamic pricing model to be used by operators over heterogeneous wireless networks and we have used Game Theory techniques so as to study the optimum strategies.

In particular, two different analysis have been carried out. On the one hand we have made a theoretical study about the expected behavior of the system in some illustrative situations. Since the complexity of the subjacent scenario might get too high, we have carried out a more realistic analysis so as to corroborate the benefits of using dynamic pricing strategies. For this purpose, we have posed a finite game, in which the players are the available AEs. The obtained results show that the use of the optimum strategies (the Nash Equilibrium of the games) brings about additional revenue to the operators, which would obtain a higher benefit than that assessed with more traditional policies. Furthermore, two complementary network deployments were analyzed and it was shown that the deterministic network deployment yields slightly better results, since it somehow reflects an optimum distribution of the AEs.

The work presented in this paper sets the basis for a wide range of research lines, some of them have already started. We will also use another approach, by means of an event-driven simulator [3] so as to see how the proposed scheme works when including both user movement and different traffic patterns. Other analysis types, such as linear programming, can also be followed in future work. In addition, we could increase the complexity of the pricing policies, by allowing the AEs to provide differentiated prices per user; besides, the developed framework might be combined with other parameters like resource allocation from the operators.

\section{Acknowledgements}

The authors would like to express their gratitude to the Spanish government for its funding in the following project: "Cognitive, Cooperative Communications and autonomous SErvice Management", C3SEM (TEC2009-14598-C02-01). The authors also acknowledge the European Commission for its funding through the "Scalable and Adaptive Internet Solutions", SAIL Project (FP7-ICT-2009-5257448)

\section{References}

1. Baliosian, J., Rubio-Loyola, J., Salazar, P., Ag R.: Gestión de políticas y precios en entornos de acceso heterogéneos. X Jornadas de Ingeniería Telemática (2011) 
2. Chen, Q.B., Zhou, W.G., Chai, R., Tang, L.: Game-theoretic approach for pricing strategy and network selection in heterogeneous wireless networks. Communications, IET 5(5), $676-682$ (25 2011)

3. Choque, J., Agüero, R., Muñoz, L.: Simulation framework for the evaluation of access selection algorithms over heterogeneous wireless networks. In: 3rd International ICST Conference on Mobile Networks and Management. MONAMI (2011)

4. McKelvey, et al.: Gambit: Software tools for game theory, version 0.2010.09.01. http://www.gambit-project.org (2010)

5. Niyato, D., Hossain, E.: Competitive pricing in heterogeneous wireless access networks: Issues and approaches. Network, IEEE 22(6), 4 -11 (november-december 2008)

6. Niyato, D., Hossain, E.: A game theoretic analysis of service competition and pricing in heterogeneous wireless access networks. Wireless Communications, IEEE Transactions on $7(12), 5150-5155$ (december 2008)

7. Sengupta, S., Anand, S., Chatterjee, M., Chandramouli, R.: Dynamic pricing for service provisioning and network selection in heterogeneous networks. Physical Communication 2(12), 138 - 150 (2009)

8. Sengupta, S., Chatterjee, M., Kwiat, K.: Pricing-based service and network selection in overlaid access networks. In: Information, Communications Signal Processing, 2007 6th International Conference on. pp. 1 -5 (dec 2007)

9. Watson, J.: Strategy, An Introduction to Game Theory. W. W. Norton \& Company, second edn. (2002) 